\title{
Development of Aloe Vera Jelly for Diabetic Patients and Analysis of Its Physicochemical Properties
}

\author{
Rebeka Sultana, Rokeya Begum*, Md. Nannur Rahman, Md. Rakibul Hasan, Md. Azizul Haque \\ Department of Food Technology and Nutritional Science, Mawlana Bhashani Science and Technology University, Santosh, Bangladesh
}

Email address:

rokeya15@yahoo.com (R. Begum)

${ }^{*}$ Corresponding author

\section{To cite this article:}

Rebeka Sultana, Rokeya Begum, Md. Nannur Rahman, Md. Rakibul Hasan, Md. Azizul Haque. Development of Aloe Vera Jelly for Diabetic Patients and Analysis of Its Physicochemical Properties. International Journal of Food Science and Biotechnology.

Vol. 5, No. 1, 2020, pp. 1-5. doi: 10.11648/j.jifsb.20200501.11

Received: November 9, 2019; Accepted: December 9, 2019; Published: January 6, 2020

\begin{abstract}
An attempt has been made in the present study to develop aloe vera jelly with honey in a view using it as an antidiabetic agent for its therapeutic properties. Different aloe vera jellies were prepared with different sweetening agents including honey, sugar and aspartame to make a comparison on the basis of nutrient content, bioactive compounds, organoleptic quality, syneresis and microbial stability. Prepared jellies were analyzed for physico-chemical properties, bioactive compound (total phenolic content, vitamin C, and antioxidant) and organoleptic properties. Among the three jellies aloe vera jelly with honey exerts better organoleptic properties, higher consumer acceptance, higher textural profile, highest amount of bio-active compounds (total phenolic content, vitamin $\mathrm{C}$ and antioxidant) and lowest syneresis. Thus formulation of aloe vera jelly with incorporation of honey increases the sensory attributes, textural profile and decrease syneresis followed by longer shelf life. Therefore, aloe vera jelly with honey could be recommended for large scale production of aloe vera jelly which can be promoted as an anti-diabetic agent due to hypoglycemic properties of aloe vera and honey.
\end{abstract}

Keywords: Aloe Vera Gel, Jelly, Antioxidant, Honey, Anti-diabetic, Syneresis

\section{Introduction}

Diabetes is a chronic disease marked by the higher level of blood glucose from defects in insulin production, insulin action or both [1]. It is among the top 10 causes of death in adults, and was estimated to have caused four million deaths globally in 2017 [2]. In 2017, global health expenditure on diabetes was estimated to be USD 727 billion [2]. Since 2000, the International Diabetes Federation (IDF) has reported the national, regional and global occurrence of diabetes. In 2009 it was estimated that 285 million people had diabetes (T1D and T2D combined) [3], increasing to 366 million in 2011 [4], 382 million in 2013 [5], 415 million in 2015 [6] and 425 million in 2017 [2]. These days great attention is being given to management of diabetes with medicinal plants along with dietary restriction. Today a great number of modern drugs are still derived from natural sources and $25 \%$ of all prescriptions contain one or more active ingredients from plants [7]. Aloe vera has marvelous medicinal properties. It contains a plenty of bioactive components, enzyme, minerals and vitamins [8]. Aloe vera lowers blood glucose levels in diabetic patients. It also improves the responsiveness of the body tissues towards insulin, thereby making insulin more effective. This makes aloe vera extremely suitable for wide scale treatment to all diabetic conditions [9].

Nowadays, using recipes of traditional medicine, including the use of apicultural products (i.e., honey), the diabetic patients can maintain the normal level of insulin in the blood and also their overall health condition. Honey composition comprises more than 200 components, with fructose, glucose, and water as main substances. Fructose content of honey varies from 21 to $43 \%$ and the fructose/glucose ratio from 0.4 to 1.6 or even higher [10]. Although fructose is the sweetest naturally occurring sweetener, it has a glycemic index of 19, compared to glucose which has 100 or sucrose (refined sugar) with 60 [11]. There is evidence that fructose tends to lower blood glucose in animal models of diabetes $[12,13]$. Mechanisms 
involved in this process may include reduced rate of intestinal absorption [14], prolongation of gastric emptying time $[15,16]$, and reduced food intake [17, 18]. Fructose stimulates glucokinase in hepatocytes, which plays an important role in the uptake and storage of glucose as glycogen by the liver. Glucose on the other hand, which is present beside fructose in honey, enhances the absorption of fructose and promotes its hepatic actions through its enhanced delivery to the liver [19]. Aloe vera and honey has been regarded as one of the most beneficial natural and herbal remedy to combat diabetes due to having lots of active components. Attempt has been taken by the authors to develop aloe vera jelly containing honey in a view working as an anti-diabetic agent and a functional food for diabetic patients.

\section{Materials and Methods}

\subsection{Raw Materials}

Aloe vera leaves, honey, sugar (sucrose) and aspartame were procured from the local market. Other chemicals e.g. citric acid and pectin were collected from laboratory. The experiments were conducted in the Department of Food Technology and Nutritional Science, Mawlana Bhashani Science and Technology University, Tangail. Bangladesh.

\subsection{Process of Aloe Vera Jelly Preparation}

Clean and washed aloe vera was peeled and gel was extracted. Then filtration was done to get clear gel. The aloe vera jelly was prepared according to standard method. Three types of aloe vera jelly were prepared by three different type sweeteners, such as sugar, honey and aspartame. The aloe vera gel, sweetener and citric acid for each jelly were mixed and cooked and poured into a jelly bottle for further analysis. Pilot experiments were carried out to standardize the materials (sugar, honey and water) and method (cooking time) for jelly preparation.

\subsection{Sensory Analysis}

The sensory evaluation of prepared aloe vera jelly was carried out by a 20 member of panelist to judge the general appearance, color, flavor, texture, taste and overall acceptability of prepared jelly. Judgments were made through rating products on a nine point Hedonic Scale with corresponding descriptive terms ranging from 9 'like extremely' to 1 'dislike extremely' [20].

\subsection{Texture Analysis}

Jelly texture profile was analyzed by two bit compression test using texture analyzer (Food Texture Analyzer FRTS series) according to Koh et al. [21] with small modification in probe size. The prepared jelly was stored at $4{ }^{\circ} \mathrm{C}$ for around 18 hours before this test was carried out. The jelly was equilibrated to room temperature before measurement. The jelly was deformed by compression at a constant speed of $2.0 \mathrm{~mm} / \mathrm{s}$ to a distance of $4 \mathrm{~mm}$ from the gel surface using a cylindrical probe having diameter of $6 \mathrm{~mm}$. All measurements were carried out in twice.

\subsection{Physicochemical Analysis}

Total titratable acidity was determined by titration with $0.1 \mathrm{~N} \mathrm{NaOH}$ [22]. A standard pH meter (OHAUS- ST 3100) was used for the determination of $\mathrm{pH}$. Syneresis of aloe vera jelly were done by using the method as described by Hassan et al. [23] $25 \mathrm{ml}$ of aloe vera jelly at $5^{\circ} \mathrm{C}$ was slowly transferred to $50 \mathrm{ml}$ capacity centrifuge tubes causing minimum disturbance to the coagulum and centrifuged at 3394 RPM in a centrifuge (Make-Remi, India) for $20 \mathrm{~min}$. The quantity of water separated at the top of the coagulum inside centrifuge tubes was recorded as milliliters. The weight fraction of the supernatant liquid was used as index of jelly syneresis.

$$
\text { Syneresis percentage }=\frac{\text { Total weight of separated liquid }(\mathrm{g})}{\text { Total weight of sample }(\mathrm{g})} \times 100
$$

\subsection{Determination of Total Phenolic Content}

Total phenolic compound was determined by the method of Singleton and Rossi [24] using Folin-Ciocalteo reagent and by measuring the absorbance of sample extract at $765 \mathrm{~nm}$ against blank solution and expressed as gallic acid equivalent (mg GAE g-1 dry weight).

\subsection{Determination of Antioxidant Activities}

The DPPH radical scavenging capacity of each sample was determined according to Miliauskas et al. [25] The mixture containing the DPPH (2, 2-diphenyl-picrylhydrazyl) turns from deep violet to a yellow solution. The higher the antioxidant activity the discoloration rate was faster. The absorption was measured using a spectrophotometer (MODEL: T60 U, S/N: 18-1650-01-1010 AC230V (50-60Hz $150 \mathrm{~W})$ at $515 \mathrm{~nm}$ which gives the per cent inhibition of the sample and the equation is;

$$
\% \text { Inhibition }=\frac{\mathrm{A}-\mathrm{B}}{\mathrm{A}} \times 100
$$

Where, $\mathrm{A}$ is the absorbance of pure DPPH in oxidized form and $\mathrm{B}$ is the absorbance of sample taken after 15 minutes of reaction with DPPH.

\subsection{Microbiological Analysis}

One gram (1g) of jelly sample was taken into a culture tube and dissolved with $9 \mathrm{~mL}$ of peptone water. Nutrient agar was used as the culture media for the determination of total plate count and incubated at $36^{\circ} \mathrm{C}$ for 48 hours. Colony counter was used for the enumeration of total plate count. Yeast count was determined by inoculating the sample on potato dextrose agar and incubated at $25^{\circ} \mathrm{C}$ for 5 days [26]. 


\section{Results and Discussions}

\subsection{Process Optimization for the Preparation of Aloe Vera Jelly Containing Honey for Diabetic Patient}

Several experimental attempts have been made in order to optimize the final procedure of jelly making. It involves experiments with different concentration of different ingredients needed for jelly preparation. Finally the most optimized ingredients with optimal concentration have been used for jelly preparation.

\subsection{Characteristic of Raw Materials}

The physicochemical properties of basic two ingredients of aloe vera jelly i.e. honey and aloe vera gel were observed.

Table 1. Chemical characteristic of raw materials.

\begin{tabular}{lll}
\hline Test & Honey & Aloe vera \\
\hline $\mathrm{pH}$ & $3.92 \pm 0.06$ & $4.65 \pm 0.04$ \\
Moisture (\%) & $13.18 \pm 0.02$ & $96.89 \pm 0.01$ \\
$\mathrm{TSS}$ & 78 & 14 \\
Ash (\%) & $0.14 \pm 0.03$ & $0.16 \pm 0.11$ \\
Protein (\%) & $0.73 \pm 0.05$ & $0.23 \pm 0.42$ \\
Vitamin-C (\%) & $2.74 \pm 0.02$ & $0.0134 \pm 0.01$ \\
\hline
\end{tabular}

\begin{tabular}{lll}
\hline Test & Honey & Aloe vera \\
\hline Acidity (\%) & $4.74 \pm 0.65$ & $0.6 \pm 0.01$ \\
Antioxidant activity (\%) & $24.34 \pm 0.05$ & $53.047 \pm 0.07$ \\
Total phenolic content $(\mathrm{mg} / \mathrm{g})$ & $7.0 \pm .31$ & $1.97 \pm 0.51$ \\
\hline
\end{tabular}

Means $\pm \mathrm{SD}$, calculated as percentage for aloe vera and honey analyzed individually in triplicate.

The moisture content of aloe vera was higher than honey. Importantly the antioxidant activity of aloe vera was more than double than that of honey, which is almost similar to the result reported by previous study Vidic et al. [27]. But the total phenolic content of honey and aloe vera found in this study was lower than the previous result reported by Vidic et al. [27].

\subsection{Sensory Evaluation}

As shown in the Table 2, data indicated the difference in the value of sensory parameter of prepared jelly. The sensory parameter of aloe vera jelly with sugar and honey were compared with standard jelly purchased from market. By sensory evaluation we can determine the color, flavor and texture and overall acceptability. It was determined by 9point Hedonic Scale. Table 2 shows the data of sensory evaluation.

Table 2. Sensory evaluation of three types of jelly.

\begin{tabular}{lllllll}
\hline Sample & General appearance & Flavor & Color & Texture & Taste & Overall acceptability \\
\hline Aloe vera jelly with honey & 6.8 & 6.8 & 7.2 & 6.8 & 6 & 7.4 \\
Aloe vera jelly with sugar & 8 & 7.4 & 8 & 7.2 & 7.2 \\
Aloe vera jelly with aspartame & 7.4 & 6.8 & 6.8 & 7.2 & 6.8 & 6.4 \\
\hline
\end{tabular}

Values are mean of twenty samples.

Aloe vera jelly prepared with honey exhibits better sensory scores regarding general appearance, flavor, color, texture and taste than the control sample. Precisely its overall acceptability is far better than the commercial brand and equal with aloe vera jelly prepared with sugar.

\subsection{Texture Profile Analysis}

Among three aloe vera jelly samples jelly with aspartame was unable to measure texture parameter as its structure was broken down after refrigeration. The textural profile parameters namely hardness, cohesiveness, springiness, gumminess, and chewiness of aloe vera jelly are presented in Table 3. The values of textural parameter of two aloe vera jelly were different. The jelly with honey had the higher mean value for all textural parameters than jelly with sugar. So, the honey effects on firmness, springiness and cohesiveness of jelly.

Table 3. Texture profile analysis parameters of aloe vera jelly with honey and aloe vera jelly with sugar.

\begin{tabular}{lll}
\hline Texture Profile & Jelly with honey & Jelly with sugar \\
\hline Hardness & $4.138 \times 10^{\wedge} 3 \mathrm{~N} / \mathrm{m}^{\wedge} 2$ & $2.546 \times 10^{\wedge} 3 \mathrm{~N} / \mathrm{m}^{\wedge} 2$ \\
Cohesiveness & 0.8846 & 0.8374 \\
Springiness & 0.8162 & 0.7282 \\
Gumminess & $3.660 \times 10^{\wedge} 3 \mathrm{~N} / \mathrm{m}^{\wedge} 2$ & $2.132 \times 10^{\wedge} 3 \mathrm{~N} / \mathrm{m}^{\wedge} 2$ \\
\hline
\end{tabular}

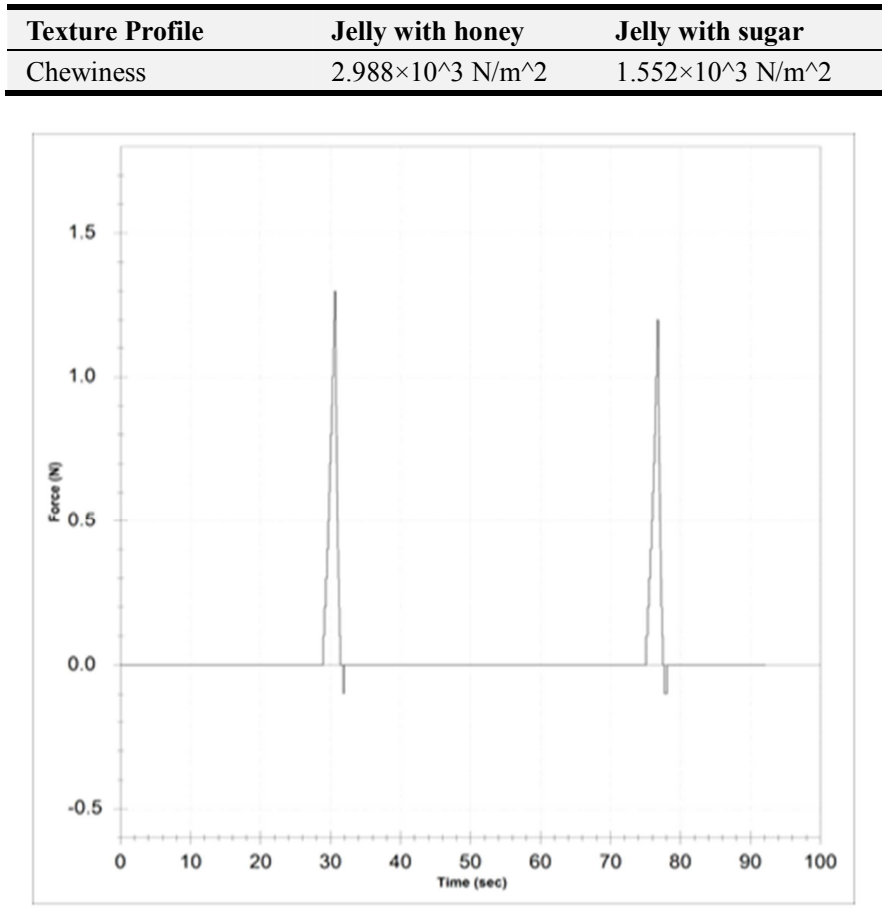

Figure 1. Texture profile of aloe vera jelly with honey. 


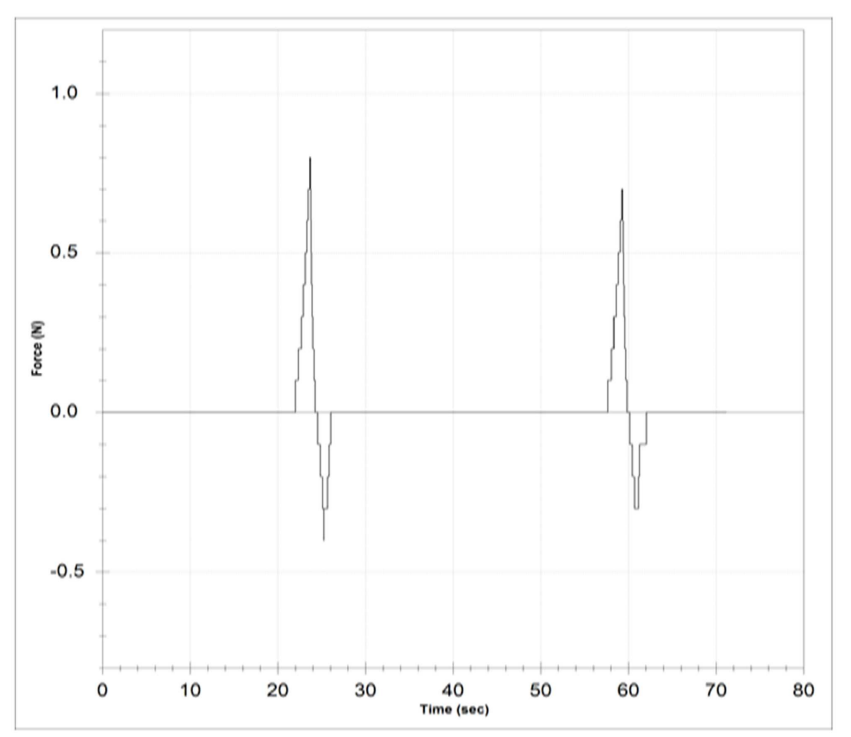

Figure 2. Texture profile of of aloe vera jelly with sugar.

\subsection{Syneresis}

Syneresis is the contraction of a gel accompanied by the separating out of liquid. After laboratory tests the percentage of syneresis of aloe vera jelly with honey, aloe vera Jelly with sugar and aloe vera Jelly with aspartame is given accordingly $7.93 \pm 1.56 \%, \quad 22.062 \pm 1.72 \%$ and $56.78 \pm 1.63 \%$. Jelly prepared with honey exhibited lowest percentage of syneresis and jelly with aspartame exhibited highest syneresis. Higher syneresis of aspartame added sample may causes growth of microorganism and spoilage of jelly.

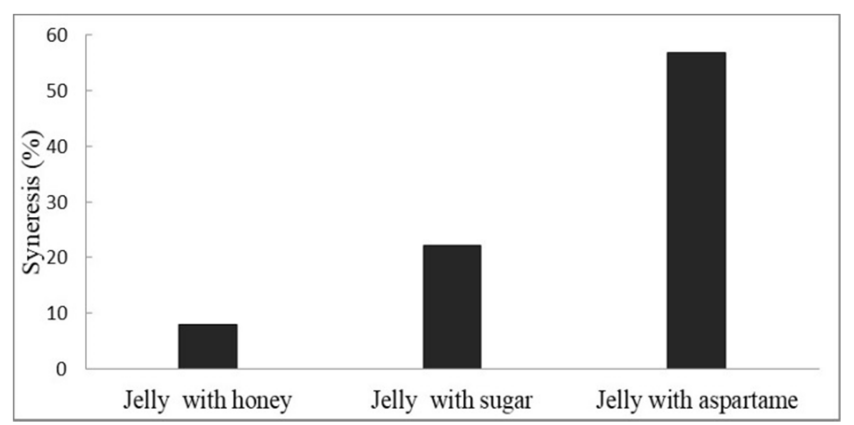

Figure 3. Syneresis percentage of aloe vera jelly.

\subsection{Microbial Analysis}

Figure 4 revealed the total viable count of bacteria and yeast colony in aloe vera jelly. After lab testing the amount of bacteria and yeast colony per gram of jelly made with aspartame was highest and jelly with honey was lowest value. The results indicate that the jelly with aspartame is more likely to contaminate by the microorganisms whereas jelly with honey has comparatively the least chance of contamination and spoilage by microorganisms. It also indicates the highest shelf life of jelly prepared with honey in comparison with two other whereas jelly with sugar indicates higher shelf life than jelly with aspartame but lower than jelly with honey.

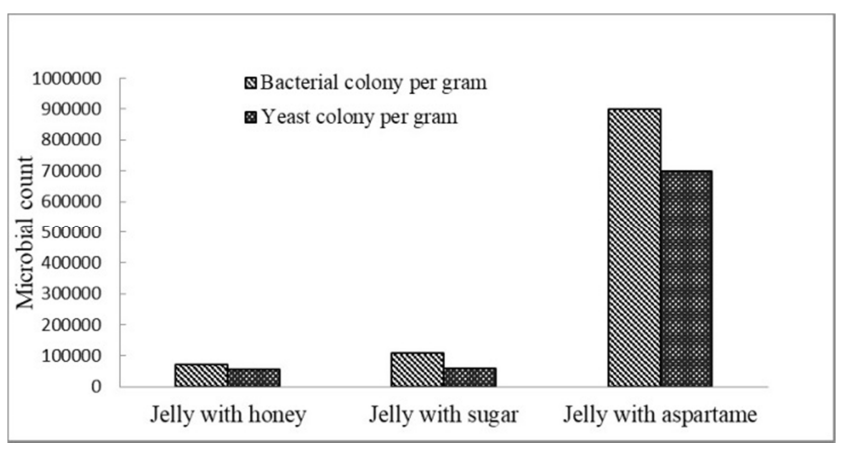

Figure 4. Microbial growth count during the $1^{\text {st }}$ day of aloe vera jelly.

\subsection{Chemical Composition of Jelly}

Table 4. Chemical properties of aloe vera jelly.

\begin{tabular}{llll}
\hline Chemical properties & $\begin{array}{l}\text { Jelly with } \\
\text { honey }\end{array}$ & $\begin{array}{l}\text { Jelly with } \\
\text { sugar }\end{array}$ & $\begin{array}{l}\text { Jelly with } \\
\text { aspartame }\end{array}$ \\
\hline Ash (\%) & $0.998 \pm 0.01$ & $0.997 \pm 0.01$ & $0.065 \pm 0.01$ \\
Moisture (\%) & $43.35 \pm 1.9$ & $22.73 \pm 0.49$ & $56.35 \pm 1.72$ \\
Vitamin-C (\%) & $0.165 \pm 0.03$ & $0.0115 \pm 0.06$ & $0.0064 \pm 0.04$ \\
Antioxidant activity (\%) & $22.04 \pm 1.56$ & $21.933 \pm 1.60$ & $21.58 \pm 1.50$ \\
Total phenolic content & $0.7 \pm 0.03$ & $0.65 \pm 0.02$ & $0.57 \pm 0.03$ \\
(mg/g) & $0.063 \pm 0.01$ & $0.053 \pm 0.04$ & $0.049 \pm 0.01$ \\
Acidity (\%) & $4.297 \pm 0.01$ & $3.293 \pm 0.03$ & $4.46 \pm 0.04$ \\
\hline pH & & & \\
\hline
\end{tabular}

Values are mean \pm SD.

The chemical properties of three different aloe vera jellies were different (Table 4). The moisture content of aloe vera jelly with aspartame was maximum whereas among three samples jelly with sugar contained lowest percentage of moisture $(22.73 \pm 0.49)$ and aloe vera jelly with honey contain slightly higher moisture than jelly with sugar (43.35 \pm 1.9$)$. Antioxidant activity and vitamin $\mathrm{C}$ content were highest in aloe vera jelly with honey $(0.165 \pm 0.03)$ and makes it more nutritious for consumption. Similarly the total phenolic content was highest in aloe vera jelly with honey $(0.7 \pm 0.03)$ in comparison with two others and obviously will render more health benefits. By laboratory test of $\mathrm{pH}$ it is evident that three types of jellies are acidic in nature. Among the three samples aloe vera jelly with honey showed better nutritional value and keeping quality.

\section{Conclusion}

Among different types of aloe vera jelly incorporated with various sweeteners, the more potential jelly to combat diabetes was put forward by analyzing different quality parameters (physico-chemical properties, bioactive compounds and organoleptic properties). Aloe vera jelly incorporated with honey had considerable effects on physicochemical and sensory properties of jelly. The comprehensive evaluation grade of aloe vera jelly with honey was excellent in terms of various quality attributes. Overall acceptability of aloe vera jelly with honey was comparatively better than other types. Similarly aloe vera jelly with honey had higher mean score in terms of textural parameters and lowest syneresis percentage than other types of jelly. Aloe 
vera jelly with honey had highest amounts of vitamin $C$, antioxidant activity, total phenolic content which renders it more beneficial for diabetic patients. It may be concluded from the study that aloe vera jelly with honey may have higher potential to act as an anti-diabetic remedy besides other treatments. But further clinical experiments are required to make it surefire and identify the precise action of aloe vera jelly with honey in preventing diabetes.

\section{References}

[1] Gupta, R., Bajpai, K. G., John, S. and Saxena, A. M. 2008. An: overview of Indian novel traditional medicinal plants with antidiabetic potentials. African J Trod Comp Alt Med 5 (1): 1-17.

[2] International Diabetes Federation. 2017. IDF Diabetes Atlas, 8th ed. Brussels, Belgium: International Diabetes Federation.

[3] International Diabetes Federation. 2009. IDF Diabetes Atlas, 4th ed. Brussels, Belgium: International Diabetes Federation.

[4] International Diabetes Federation. 2011. IDF Diabetes Atlas, 5th ed. Brussels, Belgium: International Diabetes Federation.

[5] International Diabetes Federation. 2013. IDF Diabetes Atlas, 6th ed. Brussels, Belgium: International Diabetes Federation.

[6] International Diabetes Federation. 2015. IDF Diabetes Atlas, 7th ed. Brussels, Belgium: International Diabetes Federation.

[7] Thorfeldt, S. 2005. Herbs in diabetes mellitus. Alt Med Rev 9: $13-18$.

[8] Sharrif, M. M. and Verma, S. K. 2011. Aloe vera their chemicals composition and applications: a review. Int J Biol Med Res; 2 (1): 466-71.

[9] Misawa, E., Tanaka, M., Nomaguchi, M., Yamada, M., Toida, T., Takase, M., Iwatsuki, K. and Kawada, T. 2008. Administration of phytosterols isolated from Aloe vera gel reduce visceral fat mass and improve hyperglycemia in Zucker dabetic fatty (ZDF) rats. J Obe Res Clin Prac 2: 239-245.

[10] Bobiş, O., Daniel, S., Dezmirean and Moise, A. R. (2018). Honey and Diabetes: The Importance of Natural Simple Sugars in Diet for Preventing and Treating Different Type of Diabetes. Oxidative Medicine and Cellular Longevity, Article ID 4757893, 12 pages. https://doi.org/10.1155/2018/4757893.

[11] Bantle, J. P. 2009. Dietary fructose and metabolic syndrome and diabetes. The Journal of Nutrition, vol. 139, no. 6, pp. $1263 \mathrm{~S}-1268 \mathrm{~S}$.

[12] Kwon, S., Kim, Y. J. and Kim, M. K. 2008. Effect of fructose or sucrose feeding with different levels on oral glucose tolerance test in normal and type 2 diabetic rats. Nutrition Research and Practice, vol. 2, no. 4, pp. 252-258.

[13] Erejuwa, O. O., Sulaiman, S. A. and Wahab, M. S. 2012. Fructose might contribute to the hypoglycemic effect of honey. Molecules, vol. 17, no. 12, pp. 1900-1915.
[14] Kellet, G. L., Brot-Laroche, E. and Mace, O. J. 2008. Sugar absorption in the intestine: the role of GLUT2. Annual Reviews of Nutrition, vol. 28, no. 1, pp. 35-54.

[15] Moran, T. H. and McHugh, P. R. 1981. Distinction among three sugars in their effects on gastric emptying and satiety. American Journal of Physiology Regulatory, Integrative and Comparative Physiology, vol. 241, no. 1, pp. R25-R30.

[16] Gregory, P. C., McFadyen, M. and Rayner, D. V. 1989. Relation between gastric emptying and short-term regulation of food intake in the pig. Physiology \& Bahaviour, vol. 45, no. 4, pp. 677-683.

[17] Thibault, L. 1994. Dietary carbohydrates: effects on selfselection, plasma glucose and insulin and brain indoleaminergic systems in rat. Appetite, vol. 23, no. 3, pp. 275-286.

[18] Meirelles, C. J., Oliveira, L. A., Jordao, A. A. and Navarro, A. M. 2011. Metabolic effects of the ingestion of different fructose sources in rats. Experimental and Clinical Endocrinology \& Diabetes, vol. 119, no. 4, pp. 218-220.

[19] Fujisawa, T., Riby, J. and Kretchmer, N. 1991. Intestinal absorption of fructose in the rat. Gastroenterology, vol. 101, no. 2 , pp. $360-367$.

[20] Wichchukita, S. and O'Mahonyc, M. 2014. The 9-point hedonic scale and hedonic ranking in food science: some reappraisals and alternatives. Society of Cemical Industry, doi: 10.1002/jsfa.6993.

[21] Koh, P. C., Leong, C. M. and Noranizan, M. A. 2014. Microwave-assisted extraction of pectin from jackfruit rinds using different power levels. International Food Research Journal 21 2091-2097.

[22] Caric, M., Milanovic, S. and Vucelja, D. 2000. Standard Methods for Milk and Milk Products Analysis. Novi Sad, Srbija.

[23] Hassan, L. K., Haggag, H. F., El Kalyoubi, M. H., EL-Aziz, M. A., El-Sayed, M. M. and Sayed, A. F. 2015. Physicochemical properties of yoghurt containing cress seed mucilage or guar gum. Annals of Agricultural Science; 60 (1): 21-8.

[24] Singleton, V. I. and Rossi, J. A. 1965. Calorimetri of total phenols with phosphomolybdic - phosphotungstic acid reagents. Am J Enol Viticult. 16: 144-158.

[25] Miliauskas, G., Venskotonis, P. R. and Beek, V. T. A. 2004. Screening of radical scavenging activity of some medical and aromatic plant extracts. Food Chem. 84: 231-237.

[26] Harringan, W. F. and Mccance, M. E. 1976. Laboratory methods in food microbiology. Academic Press, London.

[27] Vidic, D., Tarić, E., Alagić, J. and Maksimović, M. 2014. Determination of total phenolic content and antioxidant activity of ethanol extracts from Aloe spp. Bulletin of the Chemists and Technologists of Bosnia and Herzegovina, 42, $5-10$. 